International Mathematical Forum, 2, 2007, no. 16, 747 - 751

\title{
Complemented Subspaces in the Normed Spaces
}

\author{
H. Mazaheri and M. Nasri \\ Department of Mathematics \\ Yazd University, Yazd, Iran \\ hmazaheri@yazduni.ac.ir
}

\begin{abstract}
The purpose of this paper is to introduce and discuss the concept of orthogonality in normed spaces. A concept of orthogonality on normed linear space was introduced. We obtain some subspaces of Banach spaces which are topologically complemented.
\end{abstract}

Keywords: Banach spaces, Topologically complemented, Chebyshev subspaces, Cochebyshev subspaces

Mathematics Subject Classification: 41A65, 46B50, 46B20, 41A50.

\section{Introduction}

Suppose $X$ is a normed linear space and $x, y \in X, x$ is said to be orthogonal to $y$ and is denoted by $x \perp y$ if and only if $\|x\| \leq\|x+\alpha y\|$ for all scalar $\alpha$. If $M_{1}$ and $M_{2}$ are subsets of $X$, it is defined $M_{1} \perp M_{2}$ if and only if for all $g_{1} \in M_{1}, g_{2} \in M_{2}, g_{1} \perp g_{2}$. (see [1]). Let $M$ be a subspace of $X$, it is defined the set of the metric complemente

$$
\hat{M}=\{x \in X: \quad x \perp M\}=\{x \in X:\|x\|=\|x+M\|\}
$$

and the set of the cometric complemente

$$
\breve{M}=\{x \in X: \quad M \perp x\} .
$$

We know that a point $g_{0} \in M$ is said to be a best approximation (resp. best coapproximation) for $x \in X$ if and only if $\left\|x-g_{0}\right\|=\|x+M\|=$ $\operatorname{dist}(x, M)$ (resp. $\left.\left\|g_{0}-g\right\| \leq\|x-g\| \forall g \in M\right)$. It can be easily proved that $g_{0}$ is a best approximation (resp. best coapproximation) for $x \in X$ if and only if $x-g_{0} \in \hat{M}$ (resp. $\left.x-g_{0} \in \breve{M}\right)$. The set of all best approximations (resp. 
best coapproximations) of $x \in X$ in $M$ is shown by $P_{M}(x)\left(\right.$ resp. $\left.R_{M}(x)\right)$. In other words,

$$
P_{M}(x)=\left\{g_{0} \in M: x-g_{0} \in \hat{M}\right\}
$$

and

$$
R_{M}(x)=\left\{g_{0} \in M: x-g_{0} \in \breve{M}\right\} .
$$

If $P_{M}(x)\left(\right.$ resp. $\left.R_{M}(x)\right)$ is non-empty for every $x \in X$, then $M$ is called an Proximinal (resp. coproximinal) set. The set $M$ is Chebyshev (resp. cochebyshev) if $P_{M}(x)\left(\right.$ resp. $\left.R_{M}(x)\right)$ is a singleton set for every $x \in X$.

The problems of coapproximation was initially introduced by Franchetti and Furi ,[1], in order to study some characteristic properties of real Hilbert spaces, and such problems were considered further by Papini and Singer [7], and Rao et. al [8-12]. Also there are some results on coapproximation in [2-5]. In this context, we shall obtain some results on orthogonality and coproximinality subspaces of normed space, and obtain properties on normed spaces that are similar to orthogonality in Hilbert spaces.

Suppose $M$ is a closed subspace of a topological vector space $X$. If there exists a closed subspace $N$ of $X$ such that

$$
X=M+N \text { and } M \bigcap N=\{0\},
$$

then $M$ is said to be complemented in $X$. In this case, $X$ is said to be the direct sum of $M$ and $N$, and the notation $X=M \oplus N$. Let $M$ be a closed subspace of a topological vector space $X$. It is well known results that if $X$ is locally convex and $\operatorname{dim} M<\infty$ or $\operatorname{dim}(X / M)<\infty$, then $M$ is complemented in $X$.

In this section we shall present a list of known lemmas which are needed in the proof of main results.

Lemma 1.1. Let $X$ be a normed linear space. Let $M$ be a linear subspace of $X$, and $u \in X \backslash M$. Then the following statements are equivalent:

1) $u \in \breve{M}$

2) For all $g \in M$, there exists $f_{g} \in X^{*}$ such that $\left\|f_{g}\right\|=1, f_{g}(g)=\|g\|$ and $f_{g}(u)=0$.

Lemma 1.2. Let $X$ be a normed linear space. Let $M$ be a linear subspace of $X$, and $u \in X \backslash M$. Then the following statements are equivalent:

1) $u \in \hat{M}$.

2) There exists $f \in X^{*}$ such that $\|f\|=1, f(u)=\|u\|$ and $\left.f\right|_{M}=0$.

Lemma $1.3([4])$. Let $M$ be a closed subspace of a normed linear space $X$. Then 
i) $M \cap \breve{M}=\{0\}$.

ii) $d(g, \breve{M})=\|g\|$ for every $g \in M$.

iii) $M$ is coproximinal subspace if and only if $X=M+\breve{M}$.

iv) $M$ is cochebyshev subspace if and only if $X=M \oplus \breve{M}$, where $\oplus$ means that the sum decomposition of each element $x \in X$ is unique.

Lemma 1.4 ([13]). Let $M$ be a closed subspace of a normed linear space $X$. Then

i) $M \cap \hat{M}=\{0\}$.

iii) $M$ is proximinal subspace if and only if $X=M+\hat{M}$.

iv) $M$ is Chebyshev subspace if and only if $X=M \oplus \hat{M}$.

\section{Orthogonality complemented}

We want characterization the orthogonal complemented subspace in a normed space. In this section suppose $X$ is a normed linear space.

Definition 2.1. Suppose $M$ is a subspace of $X$ then $M$ is called orthogonal complemented subspace, if either $M$ is Chebyshev and $\hat{M}$ is a subspace of $X$ or $M$ is cochebyshev and $\breve{M}$ is a subspace of $X$.

Lemma 2.2. Let $X$ be a normed space and $M$ be a closed subspace of $X$. Then

a) $\breve{M} \perp M$ if and only if $\breve{M} \subseteq \hat{M}$, b) $M \perp \hat{M}$ if and only if $\hat{M} \subseteq \breve{M}$.

Proof. a). For all $x \in \breve{M}$, we have $M \perp x$ if and only if $\breve{M} \subseteq \hat{M}$. Also proof of b) is similar to a).

Lemma 2.3. Let $M$ be a closed subspace of an inner product space $X$ then $M^{\perp}=\hat{M}=\breve{M}$. That is, $M$ is orthogonal complemented subspace.

Theorem 2.4. Let $X$ be a smooth Banach space and $M$ be a coproximinal subspace of $X$. Then $M$ is an orthogonal complemented subspace of $X$.

Proof. Since $X$ is a smooth space, from Lemma 1.1, $\breve{M}$ is a subspace of $X$. Also $M \cap \breve{M}=\{0\}$, and $\alpha \breve{M} \subseteq \breve{M}$, therefore $M$ is cochebyshev subspace. It follows that $M$ is orthogonal complemented subspace of $X$.

Theorem 2.5. Let be a normed space and $M$ be a proximinal (resp. coproximinal) subspace of $X$. If $\hat{M}$ (resp. $\breve{M})$ is convex. Then $M$ is an orthogonal complemented.

Proof. We can prove that if $M$ is proximinal (resp. coproximinal) and 
$\hat{M}($ resp. $\breve{M})$ is convex, then $M$ is a Chebyshev (resp. cochebyshev) subspace of $X$. Therefore $M$ is orthogonal complemented.

There exist a Banach space which is not inner product space and has an orthogonal subspace.

Example 2.6. Let $\left(M_{1},\|.\|_{1}\right)$ and $\left(M_{2},\|.\|_{2}\right)$ be arbitrary Banach space. Put $X=M_{1} \oplus M_{2}$ with the norm $\|x+y\|=\|x\|_{1}+\|y\|_{2}$. We know that if $M=M_{1}$ then $\hat{M}=M_{2}$. Therefore $M$ is Chebyshev and $\hat{M}$ is subspace. It follows that $M$ is an orthogonal complemented subspace.

\section{References}

[1] G. Brikhoff, Orthogonality in linear metric space, Duke Math. J. 1(1935) 169-172.

[2] C. Franchetti, M. Furi, Some characteristic properties of real Hilbert spaces, Rwy Romaine Math., 1972.

[3] F. Mazaheri, F. M. Maalek Ghaini, Quasi-orthogonality of the best approximant sets, In press in Nonlinear Analysis, 2006.

[4] H. Mazaheri, S. M. Modarress, Some results concerning proximinality and Coproximinality, Nonlinear Analysis, 62(2005) no. 6, 1123-1126.

[5] H. Mazaheri, S. M. Vaezpour, Orthogonality and $\epsilon$-Orthogonality in Banach spaces, Aust. J. Math. ASnal. Appl., 2 (2005) no. 1, Art. 10, 1-5.

[6] T. D. Narang, Best coapproximation in metric spaces, Publ. Inst. math. (Beograd) (N.S) 51(65)(1992) 71-76.

[7] P. L. Papini, I. Singer, Best coapproximation in normed linear spaces, Mh. Math., 88(1979) 27-44.

[8] G. S. Rao, K. R. Chandrasekaran, Best coapproximation in normed linear spaces with property $(\Lambda)$, Math. Today, 2(1984) 33-40.

[9] G. S. Rao, K. R. Chandrasekaran, Some properties of the maps $R_{G}$ and $R_{G}^{\prime}$, Pure Appl. Math. Sci., 23(1986) 21-27.

[10] G. S. Rao, K. R. Chandrasekaran, Characterization of elements of best coapproximation in normed linear spaces, Pure Appl. Math. Sci., 26(1987) 139-147.

[11] G. S. Rao, R. Saravanan, Some results concerning best uniform coapproximation., Journal of Inequal. in pure and Appl. Math., 3(2002) no. 2, $1-13$.

[12] G. S. Rao, R. Saravanan, Strongly unique best coapproximation, Kyungpook math. J., 43(2003) no. 4, 519-538. 
[13] I. Singer, Best approximation in normal linear spaces by elements of linear subspaces, Springer, New York 1970.

Received: May 6, 2006 